\title{
Debulking of Advanced Gastrointestinal Stromal Tumor With Peritoneal Carcinomatosis Refractory to Imatinib and Sunitinib: A Case Report
}

\author{
Talia E. Rave ${ }^{a}$, b, d, Manrique A. Guerrero ${ }^{c}$, Derick J. Christian ${ }^{c}$, Jamshed Zuberi ${ }^{\mathrm{c}}$
}

\begin{abstract}
Gastrointestinal stromal tumors (GISTs) are non-epithelial stromal tumors that arise in the gastrointestinal tract. Pharmacological treatments for GIST are tyrosine kinase inhibitors. For metastatic disease, debulking may be helpful in reducing the tumor burden, thus increasing the effectiveness of tyrosine kinase inhibitors. Debate on whether resection would benefit the patient is still present. Here is a case of a 52-year-old African American male presenting with metastatic malignant GIST with peritoneal carcinomatosis refractory to imatinib and sunitinib. Since this patient had stage IV metastasis it was ultimately decided to proceed with a therapeutic debulking procedure. For this patient, the procedure increased the effectiveness of the medication and reduced mass effect symptoms, improving quality of life.
\end{abstract}

Keywords: Gastrointestinal stromal tumor; Debulking tumor; Peritoneal carcinomatosis; Imatinib; Sunitinib

\section{Introduction}

Gastrointestinal stromal tumors (GISTs) most commonly arise in the stomach or small intestine. They can also occur in any portion of the omentum, peritoneum and alimentary tract [1]. These tumors are rare, accounting for less than $1 \%$ of primary neoplasias of the digestive tract [2]. They arise from the proliferation of intestinal cells of Cajal, a specialized cell in the gastrointestinal tract, or the precursor of these cells [1].

About $80 \%$ of GISTs have mutations in the KIT proto-

Manuscript submitted September 30, 2020, accepted October 10, 2020

Published online December 30, 2020

aSchool of Medicine, St. George's University, Grenada, West Indies bSt. Joseph's University Medical Center, 703 Main St., Paterson, NJ 07503, USA

'Department of Surgery, Faculty of Surgery, St. Joseph's Hospital University Medical Center, Paterson, NJ, USA

${ }^{d}$ Corresponding Author: Talia E. Rave, St. Joseph's University Medical Center, 703 Main St., Paterson, NJ 07503, USA. Email: rave.talia@gmail.com

doi: https://doi.org/10.14740/jmc3598 oncogene, a receptor tyrosine kinase. A smaller subset has mutations in a related receptor tyrosine kinase, platelet-derived growth factor receptor-alpha (PDGFRA). About $12 \%$ of GISTs have neither KIT nor PDGFRA mutations [3]. Imatinib (Gleevec) treatment targets the tyrosine kinase and aims at inhibiting its function.

Patients with advanced GIST may have the option for a debulking procedure after tyrosine kinase inhibitor (TKI) therapy. Studies have demonstrated that unresectable GISTs treated with imatinib therapy can have a pathological response and become resectable tumors. Performing surgeries in these cases can lead to an improved prognosis [4].

A debulking procedure may benefit the patients with metastasis if they have a partial response to imatinib or if they have isolated sites of progression. This palliative procedure, however, is still controversial. The reason for debulking metastatic GIST is the theory that reducing the tumor burden would delay or prevent the development of resistant clones [3]. Most patients with GIST develop secondary resistance mutations in KIT leading to progressive disease [5]. In addition, the TKI therapy may be able to work more effectively given a reduction in the tumor burden, and it is recommended that these patients undergoing resection of metastatic disease should resume TKI therapy indefinitely.

Described in this case is a malignant GIST with peritoneal carcinomatosis refractory to imatinib and sunitinib that was treated with a therapeutic debulking procedure. This is reported to show a survival rate of a patient with stage IV malignant GIST after a therapeutic debulking procedure.

\section{Case Report}

A 52-year-old African American male with a past medical history of anemia of chronic disease and GIST diagnosed 2 years prior presented to the emergency department complaining of progressive diffuse sharp abdominal pain, abdominal distension, nausea, increasing weakness and fatigue for 1 week. He had a debulking partial gastrectomy 2 years prior to presentation. He was taking imatinib, which was recently switched to sunitinib. On clinical examination his abdomen had a midline abdominal scar, soft, moderate abdominal distention, moderately diffuse tenderness in all four quadrants, and mild ascites. 


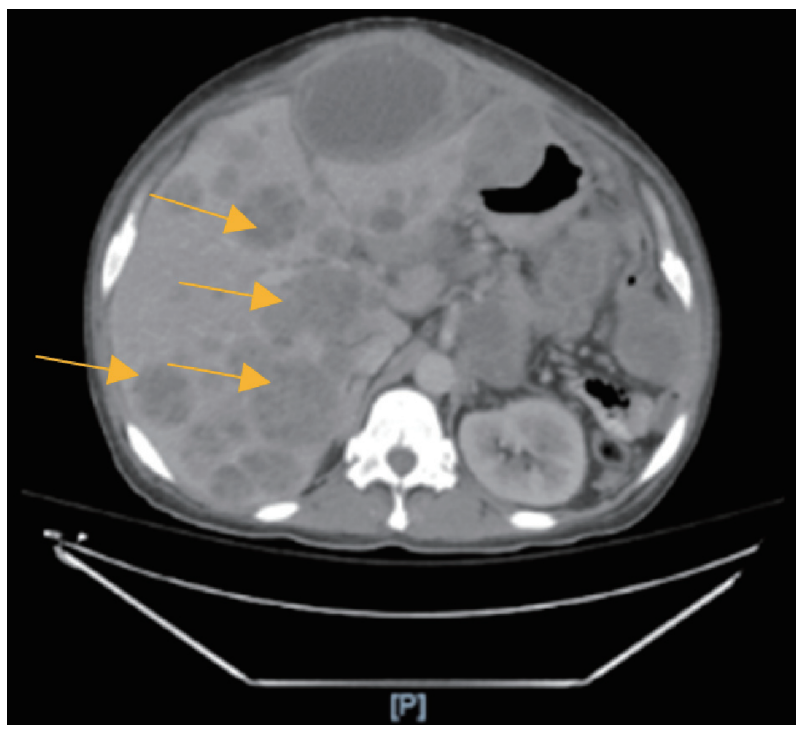

Figure 1. Computed tomography (CT) scan showing multiple necrotic liver metastasis.

The abdominal and pelvic computed tomography (CT) with contrast was compared to a recent test performed 1 month prior, which found severe metastatic disease to the liver with carcinomatosis and hepatomegaly showing mass effect on the intrahepatic vasculature and the left hepatic lobe. In addition, multiple dilated loops of small bowel without a discrete transition point suggested an ileus or early/partial small bowel obstruction (Figs. 1, 2).

The patient was admitted, and his symptoms continued to progress, including firm abdominal distension, and guarding on physical exam. The patient's cancer pathology confirmed the diagnosis as ultra-high-risk disease in the setting of c-kit positive, (PDGFRA) negative, DOG 1+ and high mitotic activity with six mitoses per high-power field.

During the admission, the patient's symptoms worsened. Progressive mass effect due to growing tumor caused restrictive lung disease and a small bowel obstruction. Intractable pain, diffuse GIST, failure to thrive and a severely distended abdomen led to an exploratory laparotomy on day 15 .

The exploratory laparotomy displayed a tumor that was adhered to multiple viscera. The tumor had grown in the liver, stomach, duodenum and the center of the abdominal cavity over the small bowel. There were multiple nodules palpated within the liver and the mesentery at its root. In total a resection of three intra-abdominal masses (colon, abdominal wall, and mesentery), a subtotal colectomy, an appendectomy, and an end ileostomy was performed. The resected tumor specimens were well-circumscribed and well vascularized.

On postoperative day (POD) 16 regorafinib (Stivarga) 160 $\mathrm{mg}$ was initiated. At this point the patient's abdominal girth had significantly decreased. He was able to tolerate an oral diet without nausea, vomiting or significant abdominal pain. The patient had a functioning ostomy postoperative and was no longer obstructed. He was able to walk around, sit up and lay in bed comfortably with a less distended abdomen. He was discharged to subacute rehab on enteral diet.

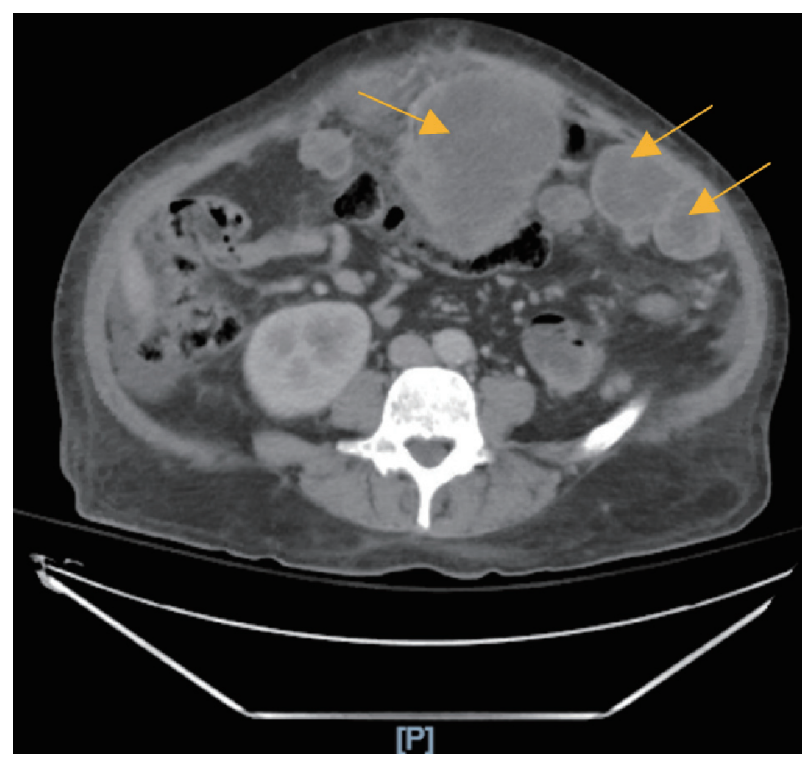

Figure 2. Computed tomography (CT) scan showing extensive carcinomatosis with multiple bulky necrotic masses.

\section{Discussion}

Imatinib was discovered in the mid-1990s to inhibit tyrosine kinase receptors C-KIT and PDGFR. It was originally used to treat chronic myelogenous leukemia, and it was not used for GIST until 1998, when Hirota et al discovered that GIST could be caused by C-KIT mutation in tyrosine kinase [1]. Today imatinib is still used as the first line treatment for GIST. If the patient is resistant and has progressive disease, sunitinib can be used. If the disease continues to progress, as it did for our patient, regorafenib can be used [6]. Regorafinib inhibits kinase receptors involved in neoplastic angiogenesis (VEGFR1-3, TEK) and neoplastic stroma (PDGFR and FGFR) [7].

Surgery is another treatment option for GIST that has been found to be successful in several cases. A study performed at Sloan-Kettering Memorial Hospital evaluated 200 patients with GIST who underwent resection. The overall survival rate was $35 \%$ at 5 years in these patients [8]. However, it is important to note that 93 patients had primary disease and $86 \%$ of them underwent a complete resection [8]. The results in this report were comparable to a study performed by $\mathrm{Ng}$ et al, which evaluated 191 patients and found a $28 \%$ survival at 5 years $[8,9]$. A surgical goal of partial resection should only be used to relieve palliative symptoms such as bleeding, pain or mass effect [8]. In our patient, the GIST had metastasized throughout the abdomen and progressed to peritoneal carcinomatosis. Therefore, a debulking procedure was performed for palliative therapy secondary to mass effect.

Other research reports found a significant difference in overall survival between patients with partial response, stable disease and progressive disease [3]. Patients with partial response and stable disease both had significantly longer overall survival than that of the group with progressive disease, with a 2 -year survival of $100 \%$ [3]. However, overall, the data provided in this study showed evidence that resection of 
metastatic GIST after TKI therapy is associated with a longer median overall survival compared with the nonsurgical cohort [3]. Patients with advanced GIST who have a response to treatment had benefited from a debulking palliative surgery [3]. In the case presented, the patient was more responsive to pharmacological therapy after the debulking procedure. This could have been due to the post-surgical reduction in the tumor burden.

Raut et al looked at patients with advanced GIST and noted that there was a prolonged overall survival after debulking procedures in these patients with stable disease or limited progression on kinase inhibitor therapy [10]. This report evaluated patients presenting with generalized progression of their disease and recorded that all patients died within 12 months of the debulking procedure. Patients with limited disease progression and generalized disease progression had a median time to death of 29.8 months and 5.6 months, respectively [10]. This study found that debulking procedures prolonged overall survival in certain patients depending on their disease progression.

Other studies found similar data supporting a debulking procedure in cases of advanced GIST. Rubio-Casadevall et al found a longer median survival in patients with advanced GIST who were responsive to imatinib therapy and underwent surgical excision of residual lesions compared to patients who received imatinib therapy alone (87.6 months versus 59.9 months) [11].

\section{Conclusions}

Overall, a debulking procedure can be effective in prolonging overall survival rate for patients with GIST who have limited progression or advanced GIST that shows response to treatment. These surgeries may also be helpful for cases of advanced GIST when used as palliative therapy, similar to our patient.

\section{Acknowledgments}

None to declare.

\section{Financial Disclosure}

This research did not receive any specific grant from funding agencies in the public, commercial, or not-for-profit sectors.

\section{Conflict of Interest}

None to declare.

\section{Informed Consent}

The manuscript has been de-identified. Informed consent was obtained from the patient.

\section{Author Contributions}

TR wrote the case report, participated in the patient care, revised and edited the case report, and approved the final version of the case report. MG participated in the patient care, revised and edited the case report, and approved the final version of the case report. DC participated in the patient care, revised and edited the case report, and approved the final version of the case report. JZ revised and edited the case report and approved the final version of the case report.

\section{Data Availability}

The authors declare that data supporting the findings of this study are available within the article.

\section{References}

1. Caram MV, Schuetze SM. Advanced or metastatic gastrointestinal stromal tumors: systemic treatment options. J Surg Oncol. 2011;104(8):888-895.

2. Miyahira CK, Bonfitto M, de Lima Farto JF, de Figueiredo Calili A, da Silva Sousa NR, de Figueiredo Calili AP. Extragastrointestinal stromal tumor: a differential diagnosis of compressive upper abdominal tumor. Case Rep Surg. 2018;2018:1052960.

3. Qiu HB, Zhou ZG, Feng XY, Liu XC, Guo J, Ma MZ, Chen YB, et al. Advanced gastrointestinal stromal tumor patients benefit from palliative surgery after tyrosine kinase inhibitors therapy. Medicine (Baltimore). 2018;97(2):e9097.

4. Fukuda S, Fujiwara Y, Wakasa T, Kitani K, Tsujie M, Yukawa M, Ohta Y, et al. Giant gastric gastrointestinal stromal tumor with severe peritoneal dissemination controlled by imatinib therapy following debulking surgery: a case report. J Med Case Rep. 2017;11(1):33.

5. Liegl B, Kepten I, Le C, Zhu M, Demetri GD, Heinrich $\mathrm{MC}$, Fletcher CD, et al. Heterogeneity of kinase inhibitor resistance mechanisms in GIST. J Pathol. 2008;216(1):6474.

6. Nishida T, Blay JY, Hirota S, Kitagawa Y, Kang YK. The standard diagnosis, treatment, and follow-up of gastrointestinal stromal tumors based on guidelines. Gastric Cancer. 2016;19(1):3-14.

7. Lugowska I, Dawidowska A, Klimczak A, Rutkowski P. Efficacy of regorafenib in gastrointestinal stromal tumours (GISTs) - case report. Oncol Clin Pract. 2017;13:177180.

8. DeMatteo RP, Lewis JJ, Leung D, Mudan SS, Woodruff JM, Brennan MF. Two hundred gastrointestinal stromal tumors: recurrence patterns and prognostic factors for survival. Ann Surg. 2000;231(1):51-58.

9. Ng EH, Pollock RE, Munsell MF, Atkinson EN, Romsdahl MM. Prognostic factors influencing survival in gastrointestinal leiomyosarcomas. Implications for surgical management and staging. Ann Surg. 1992;215(1):68-77. 
10. Raut CP, Posner M, Desai J, Morgan JA, George S, Zahrieh D, Fletcher CD, et al. Surgical management of advanced gastrointestinal stromal tumors after treatment with targeted systemic therapy using kinase inhibitors. J Clin Oncol. 2006;24(15):2325-2331.

11. Rubio-Casadevall J, Martinez-Trufero J, Garcia-Albeniz
X, Calabuig S, Lopez-Pousa A, Del Muro JG, Fra J, et al. Role of surgery in patients with recurrent, metastatic, or unresectable locally advanced gastrointestinal stromal tumors sensitive to imatinib: a retrospective analysis of the Spanish Group for Research on Sarcoma (GEIS). Ann Surg Oncol. 2015;22(9):2948-2957. 